\title{
Quality of Life Among Children with Cancer in Gaza Strip
}

\author{
Motasem Salah $^{1, *}$, Mohammed Abu Reyala ${ }^{1}$, Mohammed Al Jerjawy ${ }^{2}$ \\ ${ }^{1}$ College of Health Professions, Al Quds University, Gaza, Palestine \\ ${ }^{2}$ Palestine College of Nursing, Ministry of Health, Gaza, Palestine
}

Email address:

msalah@ucas.edu.ps (M. Salah),moh.reyala386@gmail.com (M. A. Reyala), maljerjawy2009@hotmail.com (M. A. Jerjawy)

${ }^{*}$ Corresponding author

\section{To cite this article:}

Motasem Salah, Mohammed Abu Reyala, Mohammed Al Jerjawy. Quality of Life Among Children with Cancer in Gaza Strip. American Journal of Health Research. Vol. 6, No. 5, 2018, pp. 119-125. doi: 10.11648/j.ajhr.20180605.12

Received: November 4, 2018; Accepted: November 19, 2018; Published: December 10, 2018

\begin{abstract}
The study aimed to assess quality of life among children with cancer in Gaza Strip and the factors influencing their life conditions. A descriptive, analytic, cross-sectional study include 122 children with cancer whose age was between 7 to 18 years. Data collected at El Ranteesy Specialized Pediatric Hospital and European Gaza Hospital data collected by using the Pediatric Quality of Life Inventory (PedsQL 4.0 generic core scale). Participants were interviewed by the researcher and completed the questionnaire which focused on quality of life included five domains. The result showed that most of the participants have a medium level of quality of life with the mean score was $52.53 \%$. The social function domain got the highest score with equaled $(60.98 \%)$, physical function domain $(52.94 \%)$, the school function domain $(48.85 \%)$, and the lowest domain was emotional function domain with equaled (47.13\%). Finding showed that gender, age, hospital, participants' residence, participants' father level of education, participants' mother level of education, diagnosis years for the current disease, and family history of cancer not statically significant difference in the quality of life. Providing psycho-oncology services in ministry of health together with medical treatment, anxiety reduction measurement, provide program that can enhance the level of quality of life, and psychosocial support schedule for children and their families. Finally, guiding the society of how children with cancer treated through improve the awareness and educational campaign and encourage the skills of communication for healthcare givers.
\end{abstract}

Keywords: Cancer, Quality of Life, Gaza Strip

\section{Introduction}

Cancer is considered one of the major health problems worldwide with an increasing incidence, financial load, social impact and a high mortality rate [1]. Cancer is an umbrella term used for many forms of the disease, however they all share the common formation which involves the development of uncontrolled cells, which can affect any region of the human body including organs, tissues, bones and skin [2]. Moreover, each year, more than 200,000 children are diagnosed with cancer - a disease that touches countless families and communities in all regions of the world. With access to quality care, more than $80.0 \%$ of children with cancer can survive, living full and healthy lives. However, many children in low-income and middleincome countries do not receive or complete care [2]. Furthermore, Cancer is the second leading cause of death in
Palestine, accounting for $14.0 \%$ of all death [3]. Moreover, Children with cancer accounted for $(6.5 \%)$ of the total patient with cancer in Gaza , males reported high number of children cancers it registered 119 cases which represent $(57 \%)$ of all children cancers while female cancer was registered 91 cases which represent (43\%) [4].

Though curing cancer remains the main goal of cancer research, the interest of researcher on the quality of life (QOL) assessment in chronic diseases increase constantly. There is now a great deal of information on physical, emotional and social among cancer patients, most about the period around diagnosis and the subsequence years, with better detection and treatment. People now live nearly six times longer after their cancer diagnosis than was the case 40 years ago [5].

QOL is a broad concept used to emphasize an individual's emotional reaction to life occurrences, personality, life 
fulfillment and satisfaction and with work and personal relationships, otherwise known as "well-being" [6]. Another definition encompasses the person's level of functioning and overall satisfaction and well-being of their life [7]. QOL can be influenced by many factors, including physical health and certain disease processes. One disease in particular that can significantly affect one's QOL is cancer. Cancer can be physically and psychologically wearing both during treatment and even in survivorship. In cancer survivors, QOL encompasses physical, psychological, social, and spiritual well-being [7]. Moreover, Children cancer is a major serious problem causing increase in morbidity and mortality rate in children. Internationally; child cancer is considered the second cause of death after congenital heart disease. In addition, over the past 20 years, there has been some increase in the incidence of children diagnosed with all forms of cancer; the global number of deaths from cancer will increase by nearly $80 \%$ by 2030 , with most occurring in low and middle-income countries [8].

Children with cancer accounted for $(6.5 \%)$ of the total patient with cancer in Gaza [4], this give the researcher an alarm to think deep to the consequences of that disease on the life of those children affected by it. Children Cancer in Gaza have been facing problems: such as nature of the disease, shortage of medical supply, drugs, parents' lack of awareness about the impending death of their children, the Israeli's siege that prevents patients from receiving care. In addition, unclear data for Patients' needs and lack of studies. For this reason, the current study takes place to assess the QOL of children with cancer in GS. The researcher was studied the QOL for children with cancer for the first time in Palestine.

The burden of children cancer among children increased, so it becomes a major problem with unknown causes. A great attention and efforts should be directed toward those who has disability, because those children are in desperate need of health care, rehabilitation, follow-up, health education, advising, and ongoing medical treatment, these efforts, and actions should base on careful assessments of their needs and what fits their abilities and situation, to reduces the fatigue and prepare them to live a safe and healthy life. Because QOL consider an entitlement for every person, it's a way for children happiness and civil peace, and because our children especially prove the best, and since there is no studies conducted QOL among children with cancer in our society, this effort was shed light on this subject and analyze it from many important aspects, so by this study the researcher was reached to many facts that didn't discussion about the QOL among children with cancer before, and thus access to a recommendations that being useful in planning and assurance needs of those children. The aim of this study is to assess quality of life among children with cancer in Gaza Strip and the factors influencing their life conditions.

\section{Methodology}

\subsection{Research Design}

The design of this study is descriptive, analytical and cross-sectional design. The researcher chooses to implement this design because it is the best design to describe the QOL. This type of study is useful to gather information on important health-related aspects of participants' knowledge at a specific point of time.

\subsection{Setting of the Study}

This study has been conducted at two main hospitals at El Ranteesy Specialized Pediatric Hospital and European Gaza Hospital in GS.

\subsection{The Sample}

The researcher considered the population as the sample of study, because the population size is relatively small. However, the sample of study was 122 children of the total number of the target population, there were 12 patients traveling outside GS, therefore; the researcher could not reach to them.

\subsection{Eligibility Criteria}

The study included all children between seven and 18 years old who were diagnosed with cancer and live in GS and able to response to the questionnaire. Of the total number of the target population, there were 12 patients traveling outside GS, therefore; the researcher could not reach to them.

\subsection{Study Instrument}

Pediatric Quality of Life Inventory (PedsQL 4.0 generic core scale) was used for this study to assess QOL. The PedsQL scale is interviewer-administrated QOL scoring system. The 23 - item multidimensional PedsQL 4.0 Generic Core Scales encompass 4 scales: physical functioning, emotional functioning, social functioning, and school functioning. The participants evaluated how often a specific problem occurred in the past month, A 5-point Likert response scale is utilized across child self - report for ages 7 18 years $(0=$ never a problem; $1=$ almost never a problem; 2 = sometimes a problem; $3=$ often a problem; $4=$ almost always a problem).

\subsection{Reliability and Validity of the Instrument}

The Arabic version was validated in our culture and showed high reliability with Cronbach's alpha coefficient of 0.86 [9].

\subsection{Pilot Study}

A pilot study on 16 children was done to assess the adequacy of the data collection plan, to explorer whether respondents understand the questions in the same way, to reduce the problems which may rise during data collection, to identify all domains and components of instrument and to determine the particular time needed to fill the two questionnaires. 


\subsection{Ethical Consideration and Procedures}

The ethical, administrative considerations and procedures are very important conditions in applying the research. All of the ethical procedures have to be followed perfectly without ignoring any of them. It includes, an official letter of approval was obtained from Helsinki Committee in GS, an official letter was obtained from $\mathrm{MOH}$ to conduct this study and every participant was provided with an explanatory form about the study including the purpose of the study, confidentiality of information and some instructions. The researcher assured the participant that all finding of the study would be used to guide the services providers and policy makers to improve QOL for children with cancer.

\subsection{Data Collection}

Data were collected through a face-to-face interview with each participant. At the start, all questionnaire forms were prepared, organized, and classified with serial numbers to ensure the availability of the needed information. The researcher explained the purpose of the questionnaire to the participant before obtaining consent form and during the interview, any unclear information was simplified by the researcher to ensure the exact and real answer by the participant.

\subsection{Data Analysis}

Data analysis was carried out by a statistician and the researcher. Means have been computed for the continuous numeric variables. To illustrate the main characteristics of the respondents, frequency tables have been used. Advanced statistical analysis was conducted to explore potential relationships between variables, student t-test and one way ANOVA test to examine potential relationships between the continuous variables. $\mathrm{P}$ values of $\leq 0.05$ was considered statistically significant.

\section{Results}

\subsection{Socio-Demographic Characteristics of the Study Population}

Table 1 , showed that $(54.1 \%)$ of the study participants are males, while $(45.9 \%)$ of them are females. The table also showed that the majority $(86.9 \%)$ of the study participants are receiving their care and treatment from RSPH, and the rest $(13.1 \%)$ are receiving their care from EGH. Moreover, 68 $(55.7 \%)$ of the study participants are below 9 years, $40(32.8 \%)$ are between 10 and 13 years old, while 14 (11.5\%) are between 14 and 18 years old. More than half of the study participants' $(51.7 \%)$ families have level of income below $1000 \mathrm{New}$ Israeli Shekel (NIS), (30.3\%) have level of income between 1000 and 1500 Shekel, while (18.0\%) have level of income more than 1500 Shekel. Regarding the number of family members at the participants' homes; $(48.4 \%)$ of them are living with below than 4 members, $(37.7 \%)$ are living with 5 -7 members, while $(13.9 \%)$ of them are living with more than 8 members. Moreover, the table showed that $66(54.1 \%)$ of the study participants were diagnosed with cancer since more than 2 years, $31(25.4 \%)$ of them were diagnosed with cancer since below 1 years, while $25(20.5 \%)$ were diagnosed with cancer since $1-2$ years. Additionally, the table showed that the majority $(77.0 \%)$ of the study participants do not have family history of cancer, while $(23.0 \%)$ of them do.

Table 1. Socio-Demographic characteristics of the study population.

\begin{tabular}{llll}
\hline Variables & & Number & Percentage (\%) \\
\hline \multirow{2}{*}{ Gender } & Male & 66 & 54.1 \\
& Female & 56 & 45.9 \\
& RSPH & 106 & 86.9 \\
Age groups & EGH & 16 & 13.1 \\
& Below 9 years & 68 & 55.7 \\
Families level of & 10-13 years & 40 & 32.8 \\
income & 14-18 years & 14 & 11.5 \\
& Below 1000 NIS & 63 & 51.7 \\
Number of & More than 1500 NIS & 37 & 30.3 \\
family members & Below 4 members & 59 & 18.0 \\
& $5-7$ members & 46 & 48.4 \\
& More than 8 members & 17 & 37.7 \\
Diagnosis years & 1-2 years & 31 & 13.9 \\
& More than 2 years & 25.4 \\
\multirow{2}{*}{ Family history } & Present & 28 & 20.5 \\
& Not & 94 & 54.1 \\
& Total & 122 & 23.0 \\
\hline
\end{tabular}

\subsection{Quality of Life Among Children with Cancer in the GS}

Table 2 showed the mean percentage of each QOL domain among children with cancer in the GS. The table showed that the highest QOL domain is "Social functioning" which has a score of $(60.98 \%)$ out of $100.0 \%$, followed by "Physical functioning" domain which has a score of (52.94\%). On the other hand, the QOL domain related to the "School functioning" has a score of (48.85), and the "Emotional functioning" domain has a score of $(47.13 \%)$. The total mean percentage of the QOL is $(52.53 \%)$.

Table 2. Mean percentage of each QOL domain among children with cancer in the GS.

\begin{tabular}{|c|c|c|c|}
\hline No & Area & Maximum score & Mean \% \\
\hline \multicolumn{2}{|c|}{ Physical function } & 100 & 52.94 \\
\hline 1 & It is hard for me to walk more than a couple of streets (about 100 meters) & 100 & 33.61 \\
\hline 2 & It is hard for me to run & 100 & 54.71 \\
\hline 3 & It is hard for me to do sports activities or exercise & 100 & 56.56 \\
\hline 4 & It is hard for me to lift heavy things & 100 & 52.25 \\
\hline 5 & It is hard for me to have a bath or shower by myself & 100 & 67.42 \\
\hline 6 & It is hard for me to do chores around the house & 100 & 64.96 \\
\hline 8 & I feel tired & 100 & 46.93 \\
\hline
\end{tabular}




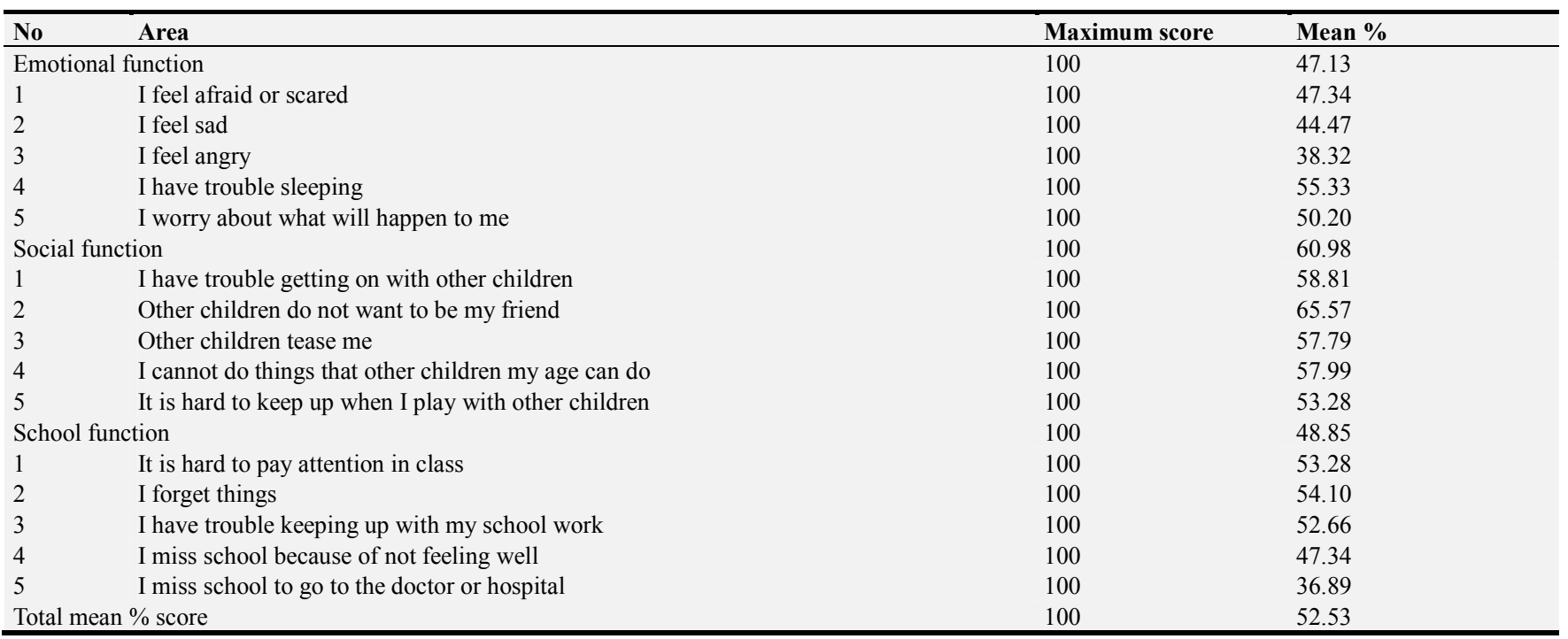

\subsection{Relationship between Study Variables and QOL Dimensions}

Table 3, showed that there is no statistically significant difference in the QOL between male and female children in the GS $(\mathrm{p}>0.05)$, Also, there is no statistically significant difference in the QOL between the children who are receiving their treatment in RSPH and who are receiving their treatment in the EGH $(p>0.05)$ and there is no statistically significant difference in the QOL among children between those who have family history of cancer and who do not $(\mathrm{p}>0.05)$.

Table 3. Relationship between all study variables and the study dimensions (Independent sample t test).

\begin{tabular}{llll}
\hline Dimension & Variable & Group & Mean (SD) \\
\hline Physical functioning & \multirow{2}{*}{ Gender } & Males & $52.56(20.70)$ \\
Emotional functioning & & Females & $52.50(17.98)$ \\
Social functioning & Hospital & RSPH & $52.05(20.13)$ \\
& & EGH & $55.77(13.83)$ \\
School functioning & Family history & Present & $50.65(19.36)$ \\
\end{tabular}

${ }^{*} \mathrm{P}$ value is significant $\leq 0.05$

Table 4 showed that there is no statistically significant difference in the QOL between different age groups of the children $(p>0.05)$. While, there is statistically significant difference in the QOL among children between their different families' level of income $(\mathrm{p}<0.05)$. Post hoc analysis was done using Scheffe test and showed that the difference is between who have family income below 1000 Shekel and 1000 - 1500 Shekel in favor of those who have family income $1000-1500$ Shekel. Also, there is statistically significant difference in the QOL among children between their different number of family members $(p<0.05)$. Post hoc analysis was done using Scheffe test and showed that the difference is between those who have family members below four and those who have more than 8 members in favor of those who have family members below 4 members. Furthermore, there is no statistically significant difference in the QOL among children between their different diagnosis years of disease diagnosis $(\mathrm{p}>0.05)$.

Table 4. Relationship between all study variables and the study dimensions (One way ANOVA).

\begin{tabular}{|c|c|c|c|c|c|c|}
\hline Dimension & Variable & Group & $\mathbf{N}$ & Mean (SD) & $F(d f)$ & $P$ value* \\
\hline \multirow{2}{*}{ Physical functioning } & \multirow{3}{*}{ Age group } & Below 9 years & 68 & $52.62(19.95)$ & \multirow{3}{*}{$1.033(2,119)$} & \multirow{3}{*}{0.359} \\
\hline & & $10-13$ years & 40 & $50.19(19.36)$ & & \\
\hline \multirow{2}{*}{ Emotional functioning } & & $14-18$ years & 14 & $58.85(16.58)$ & & \\
\hline & \multirow{3}{*}{ Families level of income } & Below 1000 Shekel & 63 & $47.55(17.85)$ & \multirow{3}{*}{$4.693(2,119)$} & \multirow{3}{*}{$0.011^{*}$} \\
\hline \multirow{2}{*}{ Social functioning } & & $1000-1500$ Shekel & 37 & $58.84(20.18)$ & & \\
\hline & & More than 1500 Shekel & 22 & $56.22(19.37)$ & & \\
\hline \multirow{2}{*}{ School functioning } & \multirow{2}{*}{$\begin{array}{l}\text { Number of family } \\
\text { members }\end{array}$} & Below 4 members & 59 & $57.64(20.01)$ & \multirow{2}{*}{$4.816(2,119)$} & \multirow{2}{*}{$0.010^{*}$} \\
\hline & & More than 8 members & 17 & $43.41(19.69)$ & & \\
\hline & \multirow{3}{*}{ Diagnosis years } & $>1$ year & 31 & $51.85(20.97)$ & \multirow{3}{*}{$0.389(2,119)$} & \multirow{3}{*}{0.678} \\
\hline Physical functioning & & $1-2$ years & 25 & $55.60(16.95)$ & & \\
\hline & & More than 2 years & 66 & $51.69(19.71)$ & & \\
\hline
\end{tabular}

\footnotetext{
${ }^{*} \mathrm{P}$ value is significant $\leq 0.05$
} 


\section{Discussion}

In this study, the result showed that $(54.1 \%)$ of the study participants are males, while $(45.9 \%)$ of them are females. It is consistent with Elnuweiry, that male was (55.5\%) while female was $(44.5 \%)$ that indicated male with cancer more than female [10]. In addition, the result was agree with Vlachioti et al., which found that $57.1 \%$ of the study participants are males, while $42.9 \%$ of them are females [11] and supported by Fawzy et al (2013) the result showed that the ratio of males to females was 1.8:1 among study patients [12].

The result of this study showed that $(55.7 \%)$ are below 9 years, $40(32.8 \%)$ are between 10 and 13 years old, while $(11.5 \%)$ are between 14 and 18 years old. The highest percentage was observed among child aged below 9 years, while the lowest was observed among child aged 14- 18 years, and the result showed that there is no statistically significant difference in the QOL between different age groups of the children, this result consistent with Al Gamal and long (2016) that there was no statistically significant differences between age of the child and total scale QOL [13], and consistent with Batalha et al (2015) indicated that statistically significant differences between the QOL and gender [14]. Here, the researcher explains that the percentage of cancer among children with cancer above 13 years is low compared with younger child on account of the nature of the disease and its complications which led to death with increasing age.

Moreover, the findings of this study revealed that $(86.9 \%)$ from children with cancer treated at RSPH. This resulted in inclusion of the highest number of children from this hospital in the study. It provides services to children from North Governorate, Gaza Governorate, and Mid-zone Governorate. The EGH is the second main hospital, which takes care of $(13.1 \%)$ of children cancer. It provide services to children from Khanyounis and Rafah Governorate. Furthermore, the study showed that there is no statistically significant difference in the QOL and HRQOL between the children who are receiving their treatment in RSPH and in EGH.

The result showed that the highest percentage was found in participants' families' level of income of below 1000 Shekel $(51.7 \%)$, while the lowest percentage was found in level of income more than 1500 Shekel. This result consisted with the study conducted by Elnuweiry (2015) that found that highest percentage $(46.6 \%)$ of cases were monthly income below1000 shekel [10]. The our result shows that there is statistically significant difference in the QOL and HRQOL among children between their different families' level of income, this result disagree with Eilertsen et al (2011) that showed that there was no statistically significant differences of children' cancer QOL in view of monthly income [15]. The researcher believed that, the economic status in the GS is very low, and suffers from continuous pressure caused by long-term siege imposed by Israelis' occupation for more than 12 years. This makes it difficult for families to provide basic health requirement for suffering children, thus, suppressing their QOL further.

Moreover, this study showed that there is no statistically significant difference in the QOL between male and female children in the GS which is inconsistent with Al-Gamal and long (2016), that indicated that boys experienced better total QOL and better physical functioning than girls [13], and consistent with Batalha et al (2015) conducted a study to describe HRQOL among children with cancer [14], and indicated that there is no statistically significant difference in the QOL and gender. Additionally, this study also showed that $(77.0 \%)$ of the study participants do not have family history of cancer, while $(23.0 \%)$ of them have family history of cancer, and there is no statistically significant difference in the QOL among children between those who have family history of disease and who do not.

The result showed that there is statistically significant difference in the QOL among children between their different number of family members. This result inconsistent with Vlachioti (2016) that there is no statistically significant difference in the QOL among children between their different number of family members [11]. The researcher believes that with a lesser number of family members, parents and caregivers can pay more attention to each individual child, and especially to a child with cancer that is in need for constant care and extra effort to improve his/her QOL.

This study showed that $(54.1 \%)$ of the study participants were diagnosed since more than 2 years, 31 (25.4\%) of them were diagnosed since below 1 years, while $25(20.5 \%)$ were diagnosed since $1-2$ years, and there is no statistically significant difference in the QOL among children between their different diagnosis years of disease, this result consistent with [11].

QOL among children with cancer

Moreover, this study showed that the total mean percentage of the QOL was $(52.53 \%)$, the social function domain got the highest score with equaled (60.98\%). Moreover, the physical function domain (52.94\%), the school function domain (48.85\%), and the lowest domain were emotional function domain with equaled (47.13).

Findings of this study are not congruent with [13] study which illustrated that the total QOL score was $(65.79 \%)$, the social domain got the highest score and the school domain score $(48.25 \%)$. Furthermore, this result is inconsistent with the study of Mounir and Abolfotouh [16] which revealed that the mean percentage of QOL score was $(62.68 \%)$, the physical domain got the highest score, then the emotional domain score $(34.4 \%)$, and the social domain (26\%). Also, findings were not confirmed by a study conducted by Rahimi et al [17] that conducted to determine related factors to QOL among children with cancer and found that the total mean percentage of QOL $(62.96 \%)$.

These results are consistent with the results of [18] which revealed that the QOL among children with cancer participating in the study score was low. Moreover, Rosenberg et al (2016) conducted a study to describe QOL 
among children with cancer and its relationship to symptom distress and showed that the total mean percentage of QOL was low level [19].

The researcher explains that cancer is considered an exhausting, and chronic nature of the disease itself in terms of frequent hospital visits, drug availability, side effects of drug and disease complication and consequence of the disease. Addition, parents' lack of awareness about the impending death of their children, and the Israeli's occupation siege that prevents patients from receiving care, all of this negatively effect on QOL and HRQOL for children with cancer in GS.

\section{Recommendation}

This study has provided useful information about QOL of children with cancer in GS. The results might help in developing deep understanding of the issues that may influence subjects' overalls health as their QOL. Furthermore, due to significant decrease in QOL domains among children with cancer in GS. I recommended to emphasize on increasing the efforts to avoid negative impacts on QOL as following: Adoption programs that can enhance the level of QOL, Psychosocial support should be schedule for children and their families, Providing psycho-oncology services in $\mathrm{MOH}$ together with medical treatment and Work collaboratively with local, regional, and national organizations related to children cancer advocacy, awareness, prevention, research, treatment, and support.

\section{Conclusion}

This study aimed to assess QOL among children with cancer who live in GS. This descriptive, analytic, crosssectional study include 122 children with cancer whose age was between 7 to 18 years. Data collected at El Ranteesy Specialized Pediatric Hospital and European Gaza Hospital. The result showed that the mean of the total QOL mean score was $(52.53 \%)$, the overall mean percentage of QOL domain scores ranged from $(47.13 \%)$ to $(60.98 \%)$. The social domain got the highest score with equaled (60.98\%) and the lowest domain were emotional function domain with equaled (47.13). Finding showed that there is statistically significant difference in the quality of life among children between their different families' level of income ( $p$ value $=0.011$ ). Moreover, the result showed that there is statistically significant difference in the quality of life among children between their different number of family members $(p$ value $=0.010$ ). The study showed that cancer has wide effects on children ability to full function in every field of their lives. It affects the physical, mental, school, and social well- being of children. The findings of the study suggest the need for support in all study dimensions for children diagnosed with cancer to minimize the disease burden. We emphasize on increasing effort to avoid negative impacts of cancer on quality of life. Providing psycho-oncology services, anxiety reduction measurement, and psychosocial support should be schedule for children and their families.

\section{Disclosures}

Authors have no conflict of interests, and the work had no financial support.

\section{References}

[1] Abed, Y, et al (2012): Cancer incidence in the Gaza Strip, Palestine (2000-2010). Cancer Registry: MOH.

[2] Word Health Organization (2017): Cancer. (http://www.who.int/mediacentre/factsheets/fs297/en/, 12.11.2017).

[3] Palestinian Health Information Center (2017): Medical Report- Nablus: $\mathrm{MOH}$, Palestine.

[4] Ministry of Health (2016): Health annual report-Ramallah, Palestine.

[5] Devance, C. (2010): Living After Diagnosis Median Cancer Survival Time, Macmillan Cancer Support.35 (4), pp. 231245 .

[6] Theofilou, P. (2013): Quality of Life: Definition and Measurement, Europe's Journal of Psychology, 9(1), pp.150-162.

[7] Lavdaniti, M, Tsitsis, N. (2015): Definitions and conceptual models of quality of life in cancer patients, Health Science Journal, 9(26), pp. 1-5.

[8] Center of Disease Control and Prevention (2014): Cancer Prevention and Control, a Global Concern. 1600 Clifton Rd. Atlanta, USA.

[9] Massad, S, et al (2011): Health-related quality of life of Palestinian preschoolers in the Gaza Strip: A cross-sectional study. BMC Public Health, 11(1).

[10] Elnuweiry, H. (2015): Risk Factors for Pediatric Cancer in the Gaza Strip, Case-Control Study. Al Quds University, Jerusalem - Palestine.

[11] Vlachioti, E, et al (2016): Assessment of quality of life in adolescent patients with cancer and adolescent survivors of childhood cancer, Journal for Specialists in Pediatric Nursing, 21(4), pp.178-188.

[12] Fawzy, M, et al (2013): Quality of Life in Egyptian Children with Cancer. Journal of Cancer Therapy, 04(07), pp.12561261.

[13] Al-Gamal, E, Long, T. (2016): Health-related quality of life and its association with self-esteem and fatigue among children diagnosed with cancer. Journal of Clinical Nursing, 25(21-22), pp.3391-3399.

[14] Batalha, L, et al (2015): Quality of life among children with cancer: agreement between child and parent reports. Esc Anna Nery, 9(2): pp. 292-296.

[15] Eilertsen, M, et al (2011): Psychosocial health in children and adolescents surviving cancer, Scandinavian Journal of Caring Sciences, 25(4), pp.725-734. 
[16] Mounir, G, Abolfotouh, M. (2007): Assessment of health related quality of life among school children with cancer in Alexandria. The Journal of the Egyptian Public Health Association, 82(3-4): pp. 219-238.

[17] Rahimi, S, et al (2014): Related factors with Quality of Life among preschool children with cancer. J Holist Nurs Midwifery; 24 (1): pp. 30-39.
[18] Nazari, B, et al (2017): A Comparison of Quality of Life, Anxiety and Depression in Children with Cancer and Healthy Children, Kermanshah-Iran. Int J Pediatric, 5(7): pp. 5305-14.

[19] Rosenberg, A, et al (2016): Quality of Life in Children with Advanced Cancer: A Report from the Pediquest Study (TH311C), Journal of Pain and Symptom Management, 51(2), p.324. 